\title{
Varying Alpha: New Constraints from Seasonal Variations
}

\author{
John D. Barrow ${ }^{1}$ and Douglas J. Shaw ${ }^{2}$ \\ ${ }^{1}$ DAMTP, Centre for Mathematical Sciences, University of Cambridge, Wilberforce Road, Cambridge CB3 0WA, UK \\ ${ }^{2}$ School of Mathematical Sciences, Queen Mary, University of London, London E1 4NS, UK
}

\begin{abstract}
We analyse the constraints obtained from new atomic clock data on the possible time variation of the fine structure 'constant' and the electron-proton mass ratio and show how they are strengthened when the seasonal variation of Sun's gravitational field at the Earth's surface is taken into account. We compare these bounds with those obtainable from tests of the Weak Equivalence Principle and high-redshift observations of quasar absorption spectra consistent with time variations in the fine structure constant.

PACS Nos: 98.80.Es, 98.80.Bp, 98.80.Cq
\end{abstract}

General relativity and the standard model of particle physics depend on at least 27 independent parameters which determine the relative strengths of the different forces, matrix angles and phases, and the masses of all known fundamental particles. These parameters are commonly referred to as the fundamental constants of Nature and we are not able to explain or predict any of their precise numerical values. This characterisation may ultimately turn out to be a misnomer, as several modern proposals for the extension of fundamental physics beyond the standard model predict that many of these parameters are neither strictly fundamental nor constant. The precise values that the 'constants' take at a given instance are often determined in terms of the vacuum expectation values of one or more scalar fields and spacetime variation of the 'constants', at some level, is therefore a prediction common to most of these theories of new physics. We also recognize that if the true fundamental theory exists in more than four spacetime dimensions then the most fundamental constants are defined there and the four-dimensional 'shadows' that we observe and call 'constants of Nature' can vary without undermining the status of constants in the higher-dimensional theory. Moreover, slow changes in the mean size of these extra dimensions will cause dimensionless constants to change at the same rate [1]. Consequently, observational investigations of the constancy of these traditional constants may play an important role in elucidating the properties of any 'Theory of Everything'.

Experimental and observational efforts to constrain the level of any possible time variation in some of these constants have a history that pre-dates the modern theories which predict how they might vary (for overviews see Refs. 2]). Until recently, all observational studies found no evidence for variations in non-gravitational constants. In the last decade, however, data from a number of astronomical observations have provided suggestions that at least two of these constants, the fine structure constant: $\alpha=e^{2} / 4 \pi \epsilon_{0} \hbar c$, and the electron-proton mass ratio: $\mu=m_{e} / m_{p}$ might have varied slightly over cosmological time. Using a data set of 128 Keck-HIRES quasar absorption systems at redshifts $0.5<z<3$, and a new many-multiplet (MM) analysis of the line separations between many pairs of atomic species possessing relativistic corrections to their fine structure, Webb et al. [3] found the observed absorption spectra to be consistent with a shift in the fine structure constant, $\alpha$, between those redshifts and the present day, of $\Delta \alpha / \alpha \equiv \alpha(z)-\alpha(0) / \alpha(0)=-0.57 \pm 0.10 \times 10^{-5} . \mathrm{A}$ smaller study of 23 VLT-UVES absorption systems between $0.4 \leq z \leq 2.3$ by Chand et al. [4] initially found $\Delta \alpha / \alpha=-0.6 \pm 0.6 \times 10^{-6}$ by using an approximate version of the full MM technique. However a recent reanalysis of the same data by Murphy et al. using the full unbiased MM method increased the uncertainties and suggested the revised figure of $\Delta \alpha / \alpha=-0.64 \pm 0.36 \times 10^{-5}$ for the same data [5]. These investigations rely on the statistical gain from large samples of quasar absorption spectra. By contrast, probes of the electron-proton mass ration can use single objects effectively. Reinhold et al. [6] found a $3.5 \sigma$ indication of a variation in the electronproton mass ratio $\mu \equiv m_{e} / m_{p r}$ over the last 12 Gyrs: $\Delta \mu / \mu=(-24.4 \pm 5.9) \times 10^{-6}$ from $H_{2}$ absorption in a different object at $z=2.8$. However, recently Murphy et al [7] have exploited the $\mu$ sensitivity of ammonia inversion transitions [8] compared to rotational transitions of $\mathrm{CO}, \mathrm{HCN}$, and $\mathrm{HCO}^{+}$in the direction of the quasar $\mathrm{B} 0218+357$ at $z=0.68466$ to yield a result that is consistent with no variation in $\mu$ when systematic errors are more fully accounted for: $\Delta \mu / \mu=\left(+0.74 \pm 0.47_{\text {stat }} \pm\right.$ $\left.0.76_{\text {system }}\right) \times 10^{-6}$, corresponding to a time variation of $\dot{\mu} / \mu=\left(-1.2 \pm 0.8_{\text {stat }} \pm 1.2_{\text {system }}\right) \times 10^{-16} \mathrm{yr}^{-1}$ in the best fit $\Lambda C D M$ cosmology.

Any variation of $\alpha$ and $\mu$ today can also be constrained by direct laboratory searches. These are performed by comparing clocks based on different atomic frequency standards over a period of months or years. Until very recently, the most stringent constraints on the temporal variation in $\alpha$ arose by combining measurements of the frequencies of $\mathrm{Sr}[9], \mathrm{Hg}+[10], \mathrm{Yb}+[11]$, and $\mathrm{H}$ [12] relative to Caesium: $\dot{\alpha} / \alpha=(-3.3 \pm 3.0) \times 10^{-16} \mathrm{yr}^{-1}$. Cingöz et al. also recently reported a less stringent limit of $\dot{\alpha} / \alpha=-(2.7 \pm 2.6) \times 10^{-15} \mathrm{yr}^{-1}[15]$; however, if the systematics can be fully understood, an ul- 
timate sensitivity of $10^{-18} \mathrm{yr}^{-1}$ is possible with their method [32]. If a linear variation in $\alpha$ is assumed then the Murphy et. al. quasar measurements equate to $\dot{\alpha} / \alpha=(6.4 \pm 1.4) \times 10^{-16} \mathrm{yr}^{-1}[3]$. If the variation is due to a light scalar field described by a theory like that of Bekenstein [13] and Sandvik, Barrow and Magueijo (BSBM) [14], then the rate of change in the constants is exponentially damped during the recent dark-energydominated era of accelerated expansion, and one typically predicts $\dot{\alpha} / \alpha=1.1 \pm 0.3 \times 10^{-16} \mathrm{yr}^{-1}$ from the Murphy et al data, which is not ruled out by the atomic clock constraints mentioned above. For comparison, the Oklo natural reactor constraints, which reflect the need for the $\mathrm{Sm}^{149}+n \rightarrow \mathrm{Sm}^{150}+\gamma$ neutron capture resonance at $97.3 \mathrm{meV}$ to have been present $1.8-2 \mathrm{Gyr}(z=0.15)$ ago, as first pointed out by Shlyakhter [16], are currently [17] $\Delta \alpha / \alpha=(-0.8 \pm 1.0) \times 10^{-8}$ or $(8.8 \pm 0.7) \times 10^{-8}$ (because of the double-valued character of the neutron capture cross-section with reactor temperature) and [18] $\Delta \alpha / \alpha>4.5 \times 10^{-8}(6 \sigma)$ when the non-thermal neutron spectrum is taken into account. However, there remain significant environmental uncertainties regarding the reactor's early history and the deductions of bounds on constants. The quoted Oklo constraints on $\alpha$ apply only when all other constants are held to be fixed. If the quark masses to vary relative to the QCD scale, the ability of Oklo to constrain variations in $\alpha$ is greatly reduced [19].

Recently, Rosenband et al. [20] measured the ratio of aluminium and mercury single-ion optical clock frequencies, $f_{\mathrm{Al}+} / f_{\mathrm{Hg}+}$, repeated over a period of about a year. From these measurements, the linear rate of change in this ratio was found to be $(-5.3 \pm 7.9) \times 10^{-17} \mathrm{yr}^{-1}$. These measurements provides the strongest limit yet on any temporal drift in the value of $\alpha: \dot{\alpha} / \alpha=(-1.6 \pm$ $2.3) \times 10^{-17} \mathrm{yr}^{-1}$. This limit is strong enough to strongly rule out theoretical explanations of the change in $\alpha$ reported by Webb et al. [3] in terms of the slow variation of an effectively massless scalar field, even allowing for the damping by cosmological acceleration, unless there is a significant new physical effect that slows the locally observed effects of changing $\alpha$ on cosmological scales (for a detailed analysis of global-local coupling of variations in constants see Refs. 21]).

It has been noted that if the 'constants' such as $\alpha$ or $\mu$ can vary, then in addition to a slow temporal drift one would also expect to see an annual modulation in their values. In many theories, the Sun perturbs the values of the constants by a factor roughly proportional to the Sun's Newtonian gravitational potential 22] (the contribution from the Earth's gravitational potential is about 14 times smaller than that of the Sun's at the Earth's surface). Hence the 'constants' depend on the distance from the Sun. Since the Earth's orbit around the Sun has a small ellipticity, the distance, $r$, between the Earth and Sun fluctuates annually, reaching a maximum at aphelion around the beginning of July and a minimum at perihelion in early January. It was shown in Ref. 23] that in many varying constant models, the values of the constants measured here on Earth, would oscillate in a similar seasonal manner. Moreover, in many cases, this seasonal fluctuation is predicted to dominate over any linear temporal drift [23].

Specifically, let us suppose that the Sun creates a distance-dependent perturbation to the measured value of a coupling constant, $\mathcal{C}$, of amplitude $\delta \ln \mathcal{C}=C(r)$. If this coupling constant is measured on the surface of another body (e.g. the Earth) which orbits the first body along an elliptical path with semi-major axis $a$, period $T_{\mathrm{p}}$, and eccentricity $e \ll 1$, then to leading order in $e$, the annual fluctuation in $\mathcal{C}, \delta \mathcal{C}_{\text {annual }}$ will be given by

$$
\frac{\delta \mathcal{C}_{\text {annual }}}{\mathcal{C}}=-c_{\mathcal{C}} \cos \left(\frac{2 \pi t}{T_{\mathrm{p}}}\right)+O\left(e^{2}\right),
$$

where $c_{\mathcal{C}} \equiv e a C^{\prime}(a), C^{\prime}(a)=d C(r) /\left.d r\right|_{r=a}$ and $t=n T_{\mathrm{p}}$, for any integer $n$, corresponds to the moment of closest approach (perihelion). In the case of the Earth moving around the Sun, over a period of 6 months from perihelion to aphelion one would therefore measure a change in the constant $\mathcal{C}$ equal to $2 c_{\mathcal{C}}$. Rosenband et

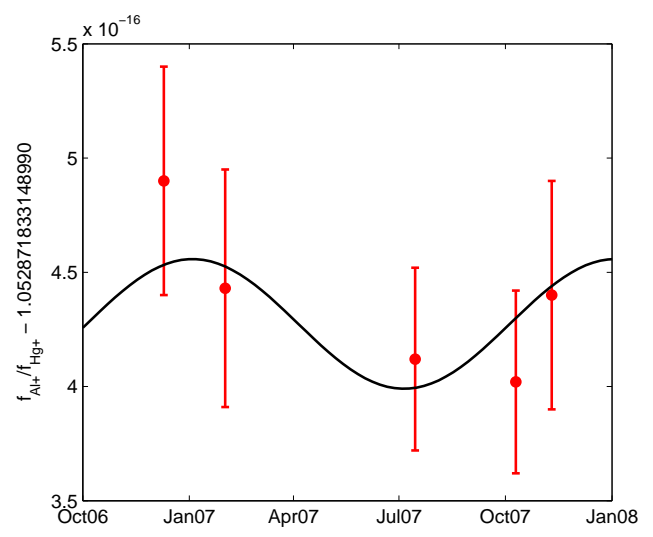

FIG. 1: (colour online). Frequency ratio $f_{\mathrm{Al}+} / f_{\mathrm{Hg}+}$ as measured by Rosenband et al. [20]. The solid black line shows the maximum likelihood fit for a seasonal variation.

al. [20] fitted a linear drift in $\alpha$ to their data finding $\dot{\alpha} / \alpha=(-1.6 \pm 2.3) \times 10^{-17} \mathrm{yr}^{-1}$. We fit the expected form of any annual fluctuation, Eq. 1, to the measured values of $f_{\mathrm{Al}+} / f_{\mathrm{Hg}+}$. The data, taken from Ref. [20], and our best fit line, are shown in Fig. 11. It should be noted that the magnitude of the systematic errors for the middle three data points were not verified to the same precision as they were from the first and last data points [20]. We do not expect to this have a great effect on the magnitude of the resulting constraint on $k_{\alpha}$. Using $\delta \ln \left(f_{\mathrm{Al}+} / f_{\mathrm{Hg}+}\right)=(3.19+0.008) \delta \alpha / \alpha,[20]$, a maximum likelihood fit to the data gives

$$
c_{\alpha}=e a \delta \alpha^{\prime}(a)=(-0.89 \pm 0.84) \times 10^{-17} .
$$


where $a=149,597,887.5 \mathrm{~km}$ is the semi-major axis of the Earth's orbit, and $\delta \alpha(r)$ is the perturbation in $\alpha$ due to the Sun's gravitational field. Assume that over solar system scales, the values of the scalar fields on which values of the 'constants' depend, vary with the local gravitational potential [22]. Hence, we have $\delta \alpha(r) / \alpha=k_{\alpha} \Delta U_{\odot}(r)$, where $k_{\alpha}$ is a theory-dependent multiplier, $\Delta U_{\odot}$ is the change in the gravitational potential of the Sun: $U_{\odot}(r)=-G M_{\odot} / r$, and so $e a \Delta U_{\odot}^{\prime}(a)=$ $e G M_{\odot} / a=1.65 \times 10^{-10}$. Hence, we find:

$$
k_{\alpha}=(-5.4 \pm 5.1) \times 10^{-8} .
$$

The frequency shifts measured by Rosenband et al. 20] were not sensitive to changes in the electron-proton mass ratio: $\mu=m_{\mathrm{e}} / m_{\mathrm{p}}$. Measurements of optical transition frequencies relative to Cs, Refs. [9, 10, 11, 12], are sensitive to both $\mu$ and $\alpha$. H-maser atomic clocks [25] are also sensitive to variations in the light quark to proton mass ratio: $q=m_{\mathrm{q}} / m_{\mathrm{p}}$. We can use all these observations if we define two more gravitational coupling multipliers, $k_{\mu}$ and $k_{\mathrm{a}}$, by $\delta \mu / \mu=k_{\mu} \Delta U_{\odot}$, and $\delta q / q=k_{\mathrm{q}} \Delta U_{\odot}$. Refs. [9, 10, 25] give $k_{\alpha}+0.36 k_{\mu}=(-2.1 \pm 3.2) \times 10^{-6}$, $k_{\alpha}+0.17 k_{\mu}=(3.5 \pm 6.0) \times 10^{-7}$, and $k_{\alpha}+0.13 k_{\mathrm{q}}=$ $(1 \pm 17) \times 10^{-7}$ respectively. We also performed a bootstrap seasonal fluctuation fit (with $10^{5}$ resamplings) to the $\mathrm{Yb}^{+}$frequency measurements of Peik et al. [11, 24] giving $k_{\alpha}+0.51 k_{\mu}=(7.1 \pm 3.4) \times 10^{-6}$. Combining these bounds with Eq. (3) gives:

$$
\begin{aligned}
& k_{\mu}=(3.9 \pm 3.1) \times 10^{-6}, \\
& k_{\mathrm{q}}=(0.1 \pm 1.4) \times 10^{-5} .
\end{aligned}
$$

Recently, Blatt et al. 9] combined data from measurements of H-maser [25] and optical atomic clocks [9, 10], to bound the multipliers, $k_{\alpha}, k_{\mu}$ and $k_{q}$, finding:

$$
\begin{aligned}
k_{\alpha} & =(2.5 \pm 3.1) \times 10^{-6}, \\
k_{\mu} & =(-1.3 \pm 1.7) \times 10^{-5}, \\
k_{\mathrm{q}} & =(-1.9 \pm 2.7) \times 10^{-5} .
\end{aligned}
$$

The constraint on $k_{\alpha}$ derived in this paper from the data of Rosenband et al. 20] therefore represents an improvement by about two orders of magnitude over the previous best bound. This improved bound on $k_{\alpha}$ combined with data found by Peik et al. [11, 24] has also produced an order of magnitude improvement in the determination of $k_{\mu}$ and a slight improvement in the constraint on $k_{q}$.

Seasonal fluctuations are predicted by a varying constant theory because the scalar field which drives the variation in the constant couples to normal matter. The presence of the Sun therefore induces gradients in scalar field and the 'constants', and it is essentially these gradients that are detectable as seasonal variables. However, as is well known, gradients in a scalar field which couples to normal matter result in new or 'fifth' forces with pseudo-gravitational effects. In the case of varying $\alpha$ and $\mu$ theories, these forces are almost always composition dependent, which would violate the universality of free-fall and hence the weak equivalence principle (WEP). The magnitude of any composition-dependent fifth force toward the Sun is currently constrained to be no stronger than $10^{-12}-10^{-13}$ times than the gravitational force 26]. In the context of a given theory the constraints from WEP tests indirectly bound $k_{\alpha}$. Indeed, they often provide the tightest constraints on $k_{\alpha}$ 23, 28, 29].

A recent and thorough analysis of the WEP violation constraints on $k_{\alpha}[28,30]$ found:

$$
k_{\alpha}=(0.3 \pm 1.7) \times 10^{-9},
$$

with a similar constraint on $k_{q}$. It must be noted, however, that this result is still subject to theoretical uncertainty, especially regarding the dependence of nuclear properties on quark masses. For instance, it was also noted in Ref. 28] that if certain (fairly reasonable) assumptions about nuclear structure are dropped, the $1 \sigma$ error bars on $k_{\alpha}$ increase by about an order of magnitude to: $\pm 1.4 \times 10^{-8}$. Despite these uncertainties, for many theories of varying $\alpha$, WEP violation constraints from laboratory experiments or lunar laser ranging 27] still provide the strongest, albeit indirect, bound on $k_{\alpha}$.

We have shown above that direct constraints on any change in $\alpha$ that is proportional to the local gravitational potential (as in general theories of its spacetime variation 22]) are now within less than 1.5 orders of magnitude of those extrapolated, under certain fairly reasonable assumptions, from the non-detection of any WEP violation. One might wonder how much further the sensitivity of atomic clock experiments to variations in $\alpha$ would have to improve before direct constraints on $k_{\alpha}$ would surpass those coming from the current WEP violation bounds. Suppose that over a few days, the ratio of two transition frequencies, $f_{\mathrm{A}} / f_{\mathrm{B}}$, can be measured with a sensitivity $\sigma_{\mathrm{f}}$, and that $\delta \ln \left(f_{\mathrm{A}} / f_{\mathrm{B}}\right)=S_{\alpha} \delta \alpha / \alpha$ (typically $S_{\alpha} \sim \mathcal{O}(1)$, although some transitions exhibit a greatly increased sensitivity [31]). The sensitivity to changes in $\alpha$ is then given by $\sigma_{\alpha}=\sigma_{\mathrm{f}} / S_{\alpha}$. By simulating data sets, we found that the sensitivity to $k_{\alpha}$ is significantly improved if one makes $N_{\mathrm{m}} \gtrsim 12$ measurements per year (at roughly regular intervals). With $N_{\mathrm{m}} \gtrsim 12$, by performing a bootstrap linear regression with $10^{5}$ resamplings of the simulated data points, we find that the sensitivity, $\sigma_{k}$ to $k_{\alpha}$ is roughly:

$$
\sigma_{\mathrm{k}} \approx 0.69 \times 10^{10} \frac{\sigma_{\alpha}}{\sqrt{N_{\mathrm{y}}\left(N_{\mathrm{m}}-1\right)}} .
$$

where $N_{\mathrm{y}}$ is the number of years for which data is taken. The total number of measurements is therefore $N_{\mathrm{y}} N_{\mathrm{m}}$. Indirect constraints currently have a sensitivity of $\sigma_{\mathrm{k}}=1.7 \times 10^{-9}[28]$. This would be surpassed by direct measurements if $\sigma_{\alpha}<2.5 \sqrt{N_{\mathrm{y}}\left(N_{\mathrm{m}}-1\right)} \times 10^{-19}$. For example, if we take measurements every 20 days (or so) over 
a single year $\left(N_{\mathrm{m}}=18, N_{\mathrm{y}}=1\right)$ then we would require $\sigma_{\alpha} \lesssim 10^{-18}$. The experiment conducted by Rosenband et al. 20] currently has a $\sigma_{\mathrm{f}} \approx 5 \times 10^{-17}$ and $S_{\alpha}=3.2$, so $\sigma_{\alpha} \approx 1.6 \times 10^{-17}$; one would therefore require an unrealistic number of measurements $N_{\mathrm{y}}\left(N_{\mathrm{m}}-1\right) \geq 4100$ to achieve the desired sensitivity to $k_{\alpha}$. Cingöz et al. obtained constraints on $k_{\alpha}$ by monitoring the transition frequencies between nearly degenerate, opposite-parity levels in two isotopes of atomic Dysprosium [15]. Currently sensitively to frequency changes is $7.5 \mathrm{~Hz}$, giving $\sigma_{\alpha}=5 \times 10^{-15}$. However, an ultimate sensitivity of about a $\mathrm{mHz}$ is predicted to be achievable [32]. This would give $\sigma_{\alpha} \approx 7 \times 10^{-19}$ and one would only require $N_{\mathrm{y}}\left(N_{\mathrm{m}}-1\right) \gtrsim 8$ to surpass the current WEP violation constraints on $k_{\alpha}$. In addition, Flambaum noted in Ref. 31] that the transition between the ground and the first excited states in the ${ }^{229} \mathrm{Th}$ nucleus is particularly sensitive to changes in $\alpha$ and $\mu$ with $S_{\alpha} \sim 10^{5}$. By measuring this transition frequency relative to, say, a Cs frequency standard, and without any improvement in measurement accuracy over what has currently been achieved, it may be possible bring $\sigma_{\alpha}$ down to about $10^{-20}$, giving $\sigma_{\mathrm{k}} \lesssim 2 \times 10^{-11}$ with $N_{\mathrm{m}} \gtrsim 12-$ two orders of magnitude better than the current WEP violation constraints. The motivation for a future space-based test of the WEP with possible sensitivity of order $10^{-15}-10^{-18}$ therefore remains very great [33, 34].

In summary: we have shown how new laboratory constraints on possible time variation in the fine structure 'constant' and the electron-proton mass ratio can yield more sensitive limits by incorporating the effects of the seasonal variation of the Sun's gravitational field at the Earth's surface. This seasonal variation is expected in all theories which require that the covariant d'Alembertian of any scalar field driving variation of a 'constant' is proportional to the dominant local source of gravitational potential [29]. The recent experimental results from Rosenband et al [20] and Peik et al, [11] have reached the sensitivity of the quasar observations of varying $\alpha$ and $\mu$ made at high redshift and we have shown may soon provide stronger bounds on varying constants than conventional ground-based WEP experiments.

Acknowledgements: DJS is supported by STFC. JDB would like to thank Paolo Molaro for discussions and for drawing attention to Ref. [20]. We are grateful to Thomas Dent and Ekkehard Peik for helpful discussions and for providing additional information regarding Refs. [28] and [1] respectively.

[1] W.J. Marciano, Phys. Rev. Lett. 52, 489 (1984); J.D. Barrow, Phys. Rev. D 35, 1805 (1987).

[2] J.D. Barrow, The Constants of Nature, Jonathan Cape,
London (2002); J-P. Uzan, Rev. Mod. Phys. 75, 403 (2003); K.A. Olive and Y-Z. Qian, Physics Today p. 4045, Oct. issue (2004); J.D. Barrow, Phil. Trans. Roy. Soc. Lond. A 363, 2139 (2005);

[3] J.K. Webb et al., Phys. Rev. Lett. 82, 884 (1999); 87, 091301 (2001).

[4] H. Chand et al., Astron. Astrophys. 417, 853 (2004); R. Srianand et al., Phys. Rev. Lett. 92, 121302 (2004).

[5] M. T. Murphy et al., Mon. Not. R. astron. Soc. 3841053 (2008); Phys. Rev. Lett. 99, 239001, (2007).

[6] E. Reinhold et al., Phys. Rev. Lett. 96, 151101 (2006).

[7] M.T. Murphy, V.V. Flambaum, S. Muller and C. Henkel, astro-ph/0806.3081v2

[8] V.V. Flambaum and M.G. Kozlov, Phys. Rev. Lett. 98, 240801 (2007)

[9] S. Blatt et al., Phys. Rev. Lett. 100, 140801 (2008).

[10] T. M. Fortier et al., Phys. Rev. Lett. 98, 070801 (2007).

[11] E. Peik et al., Phys. Rev. Lett. 93, 170801 (2004); E. Peik et al., to appear in Proceedings of the 11th Marcel Grossmann Meeting, Berlin, 2006, ed. R. Jantzen, World Scientific: Singapore, (2008), arXiv:physics/0611088.

[12] M. Fischer et al., Phys. Rev. Lett. 92, 230802 (2004).

[13] J.D. Bekenstein, Phys. Rev. D 25, 1527 (1982).

[14] H. Sandvik, J.D. Barrow and J. Magueijo, Phys. Rev. Lett. 88, 031302 (2002).

[15] A. Cingöz et al., Phys. Rev. Lett. 98, 040801 (2007).

[16] A. Shlyakhter, Nature 264, 340 (1976).

[17] Y. Fujii et al, Nucl. Phys. B 573, 377 (2000)

[18] S. Lamoureaux, Phys. Rev. D 69, 21701 (2004)

[19] V. V. Flambaum, in Proceedings of the Festschrift in Honor of Bruce H J McKellar and Girish C Joshi, Melbourne, 2006, ed. R. R. Volkas, B. H. J. McKellar, G. C. Joshi, World Scientific: Singapore, (2007).

[20] T. Rosenband et al., Science 319, 1808 (2008).

[21] D.J. Shaw and J.D. Barrow, Phys. Rev. D 71, 063525 (2005); Phys. Rev. D 73, 123505 (2006); Phys. Rev. D 73, 123506 (2006). Phys. Lett. B 639, 5962006 (2006).

[22] J. Magueijo, J.D. Barrow and H. Sandvik, Phys. Lett. B 549, 284 (2002). J.D. Barrow, H. Sandvik and J. Magueijo, Phys. Rev. D 65, 063504 (2002). J.D. Barrow and D.F. Mota, Class. Quantum Gravity, 19, 6197 (2002); J.D. Barrow, J. Magueijo and H. Sandvik, Phys. Lett. B 541, 201 (2002);

[23] D. J. Shaw (2007), arXiv:gr-qc/0702090

[24] E. Peik, [Private Communication].

[25] N. Ashby et al., Phys. Rev. Lett. 98, 070802 (2007).

[26] S. Schlamminger et al., Phys. Rev. Lett. 100, 041101 (2008); S. Baessler et al., Phys. Rev. Lett. 833585 (1999); Y. Su et al., Phys. Rev. D 50, 3614 (1994); V. G. Braginsky and V. I. Panov, Sov. Phys. JETP 34, 463 (1972).

[27] K. Nordtvedt, Phys. Rev. D 169, 1014 and 1017 (1968); D.F. Bartlett and D. van Buren, Phys. Rev. Lett. 57, 21 (1986).

[28] T. Dent (2008), arXiv:0805.0318

[29] J.D. Barrow and J. Magueijo, Phys. Rev. D 72, 043521 (2005); J.D. Barrow, Phys. Rev. D 71, 083520 (2005).

[30] T. Dent (2008), [Private Communication].

[31] V. V. Flambaum, Phys. Rev. Lett. 97, 092502 (2006); V. V. Flambaum, Phys. Rev. A 73034101 (2006).

[32] A. T. Nguyen et al., Phys. Rev. A 69, 022105 (2004).

[33] P. Touboul et al., Acta Astronaut. 50, 443 (2002).

[34] T.J. Sumner et al, Adv. in Space Research, 39, 254 (2007). 